\title{
Philosophiques
}

Chauviré, Christiane, L'oeil mathématique, Essai sur la philosophie mathématique de Peirce, Paris, Kimé, 2008, 288 p.

\section{Jérôme Havenel}

Volume 37, numéro 1, printemps 2010

Albert Lautman, philosophe des mathématiques

URI : https://id.erudit.org/iderudit/039729ar

DOI : https://doi.org/10.7202/039729ar

Aller au sommaire du numéro

Éditeur(s)

Société de philosophie du Québec

ISSN

0316-2923 (imprimé)

1492-1391 (numérique)

Découvrir la revue

Citer ce compte rendu

Havenel, J. (2010). Compte rendu de [Chauviré, Christiane, L'oeil mathématique,

Essai sur la philosophie mathématique de Peirce, Paris, Kimé, 2008, 288 p.]

Philosophiques, 37(1), 254-262. https://doi.org/10.7202/039729ar d'utilisation que vous pouvez consulter en ligne.

https://apropos.erudit.org/fr/usagers/politique-dutilisation/ 
publicité, lorsque le publicitaire se décharge de toute responsabilité en invoquant les directives de ses supérieurs et le caractère légal des procédés employés lorsqu'il collabore à une campagne éthiquement critiquable ? Cette parade commode pour les rédacteurs des poncifs commerciaux est dénoncée par l'auteur, qui rappelle à juste titre que: "Comme les autres citoyens, les publicitaires sont donc soumis absolument à la loi. Mais tout ça, c'est du droit. Redisons-le encore une fois: la loi n'est pas l'éthique» (p. 49). Il est par ailleurs d'autant plus facile de respecter la loi lorsque celle-ci est à peu près muette sur le sujet et, pour cette raison, Cossette plaide en faveur d'une certaine réglementation de la publicité. S'il est discutable de réduire, tel que le fait l'auteur, la notion d'espace public avec l'espace médiatique, c'est avec raison qu'il souligne que "[l]'espace public est un espace commun et c'est pourquoi quand l'État en balise l'accès, c'est sous certaines conditions. Or, la publicité, elle aussi, utilise l'espace public. C'est normal que la société lui concède le droit d'y accéder, mais ce serait également acceptable qu'elle le fasse en imposant un minimum de conditions assurant également le respect des droits collectifs» (p. 81).

Bref, Éthique et publicité aborde des problématiques intéressantes, et on souhaite que la démarche n'en reste pas là, puisque les sections courtes risquent de laisser certains lecteurs sur leur faim. Il n'en demeure pas moins que cet ouvrage présente une synthèse utile sur un phénomène incontournable, à savoir cette "forme d'art la plus répandue, la plus connue et la plus prisée du grand public» (p. 119) qui nous affecte tous quotidiennement. Le propos du livre nous invite à condamner, critiquer et apprécier selon le cas ces messages qui nous entourent, et surtout, à exiger que ces messages respectent notre humanité. L'éthique en publicité n'est pas que l'affaire des publicitaires: elle engage la société tout entière.

MÉLISSA THÉRIAULT

Cégep de Rimouski

\section{Chauviré, Christiane, L'œil mathématique, Essai sur la philosophie mathématique de Peirce, Paris, Kimé, 2008, 288 p.}

Ce livre reprend, pour l'essentiel, la thèse de doctorat défendue par Christiane Chauviré en 1988. L'objectif de cette étude est d'exposer la philosophie mathématique de Peirce et de la situer par rapport à ses concurrentes. L'ouvrage est divisé en trois parties. Dans la première partie, «Schématisme et analycité », l'auteure soutient qu'il faut distinguer deux concepts d'analycité chez Peirce, l'un logique, et l'autre épistémique. Dans la deuxième partie, "Logicisation de l'analytique ", elle expose les critiques de Peirce contre les insuffisances de la conception kantienne de l'analytique, puis elle soutient que la logique développée par Peirce permet d'évaluer les possibilités et les limites des machines logiques. Enfin, dans la troisième et dernière partie, 
«L'analycité et le logicisme», elle soutient qu'il y a nettement plus de divergences que de proximités entre le logicisme et la philosophie de Peirce.

L'auteure soutient que, malgré de nombreux reproches à Kant, le cœur de la philosophie des mathématiques de Peirce consiste à dire que les mathématiques ne sont ni des vérités analytiques nécessaires mais vides, ni des généralisations empiriques synthétiques mais incertaines, mais des vérités nécessaires découvertes par observations et expérimentations sur des diagrammes imaginaires. L'ouvrage de Christiane Chauviré s'intitule L'œil mathématique, Essai sur la philosophie mathématique de Peirce, car elle veut insister sur le fait que, pour Peirce, les mathématiques sont une science d'observation, ce qui veut dire que les mathématiques sont une science où l'on élabore des hypothèses, et où l'on fait des observations et des expérimentations sur ces hypothèses.

Christiane Chauviré explique que, pour Peirce, le raisonnement du mathématicien créateur n'a rien de mécanique: «l'observation $[y]$ est nécessaire, et une machine ne peut effectuer aucune inférence avant que les prémisses n'aient été préparées pour cela par cet exercice d'observation» (NEM $4.10)^{5}$. C'est pourquoi Peirce ne croit guère à la possibilité de construire des machines pensantes, même s'il a écrit à Marquand qu'il croit possible de construire " une machine pour des problèmes mathématiques réellement difficiles" (NEM 3.632). Il y a là une thèse fondamentale de la logique de Peirce qui soutient que «toute déduction implique l'observation d'un diagramme (optique, tactile ou acoustique) » (NEM 3.869, 1909).

L'auteure expose très clairement la critique que fait Peirce de la représentation mécaniste de la déduction telle qu'elle est comprise par la logique traditionnelle, dans l'ignorance de la logique polyadique, et qui, selon lui, conduit Kant à soutenir une conception insuffisante et trop étroite de l'analycité. Reprenant à sa manière le vieux problème de l'inférence immédiate, Peirce soutient qu'une déduction peut n'avoir qu'une seule prémisse, éventuellement itérée, comme lorsque de «tout homme est le fils d'un homme» on déduit que «tout homme est un petit-fils»(CP 2.469, n. 2) .

Tout en critiquant les interprétations que Hintikka fait de Kant et de Peirce, Christiane Chauviré épouse sa conception selon laquelle le kantisme en philosophie des mathématiques est une voie d'avenir si on sait séparer le bon grain de l'ivraie. Comme le montre l'auteure, Peirce approfondit le schématisme kantien pour soutenir que la pensée mathématique réside dans la construction et la transformation de diagrammes. Selon Peirce, bien que le diagramme mental imaginé puisse être actualisé par des graphes, dessins ou équations, un diagramme, c'est d'abord un signe qui représente aux yeux

5. Abrégé pour Peirce, C.S., The New Elements of Mathematics, Humanities Press, Atlantic Highlands, 1976. Cité par numéro de tome et page.

6. Abrégé pour Peirce, C.S., The Collected Papers of Charles Sanders Peirce, Cambridge, Harvard University Press, 1932 (cité par numéro de tome et de section). 
de notre esprit les objets et les relations qui se conforment à nos hypothèses.

Ainsi, le raisonnement mathématique a certes besoin de symboles pour traiter la généralité, mais il a aussi besoin d'icônes (sous la forme de constructions diagrammatiques qui font des mathématiques une science d'observation), ainsi que d'indices pour désigner ce dont il est question (les lettres indiquant les objets dont use constamment le mathématicien). Comme le rappelle l'auteure, après avoir rappelé la distinction médiévale entre les termes individuels de première intention qui dénotent des individus désignés, et les termes individuels de seconde intention qui dénotent des individus vagues, Peirce soutient que "les lettres qu'emploie le mathématicien (que ce soit en algèbre ou en géométrie) sont de tels individus par seconde intention" (CP 3.94). C. Chauviré soutient de manière convaincante que la conception de la variable chez Peirce est comparable à celle de Russell, pour lequel le " $\mathrm{X}$ » quelconque correspond à la désignation d'individus vagues dans la quantification, alors que Frege, en revanche, soutient que le « $x$ » ne peut pas dénoter un objet indéterminé, car c'est la façon de désigner la variable qui est ambiguë, l'objet étant toujours déterminé.

Christiane Chauviré souligne que, souvent, dans le cours de la démonstration mathématique, afin de traduire la formulation abstraite initiale du théorème à démontrer, on commence avec des termes singuliers (indices), puis on trace un diagramme (icône) où figurent des lettres dénotant les mêmes objets que ces termes singuliers. Avec ce procédé «la généralité [...] n'est ni perdue ni réduite, puisque l'on comprend que la lettre peut être considérée comme le nom de n'importe lequel des objets que l'étudiant peut sélectionner» (CP 4.616).

L'auteure traite de manière stimulante la question du platonisme mathématique en soutenant que chez Peirce les objets mathématiques sont créés par le mathématicien. La comparaison avec Frege est réussie et éclairante, mais on reste sur notre faim concernant celle avec des penseurs plus contemporains comme Hilbert, Gödel, Bernays, Putnam ou Kreisel. Bien qu'elle distingue les versants ontologiques et épistémologiques de la question, elle n'explique pas assez en quoi la position de Peirce est une alternative pertinente au débat entre platonisme et intuitionnisme, grâce à sa conception de l'objectivité des formes mathématiques qui correspond à ce qu'on appelle aujourd'hui le structuralisme modal, en insistant sur le type d'activité sémiotique par laquelle les structures mathématiques sont étudiées.

Christiane Chauviré rappelle la distinction faite par Peirce entre l'abstraction préscisive ${ }^{7}$, ou le fait de fixer son attention sur un aspect au détriment des autres, et l'abstraction hypostatique, par laquelle on crée des entia rationis «qui sont néanmoins parfois réels » (CP 4.549, 1906). La collection

7. Pour l'orthographe, voir André De Tienne, L'analytique de la représentation chez Peirce, Bruxelles, Publications des Facultés universitaires Saint-Louis, 1996, p. 204. 
est un exemple important d'abstraction hypostatique où est construit et créé un nouveau concept mathématique qui permet notamment de traiter comme un individu une opération (par exemple une fonction) sur des individus, et qui est, selon Peirce, essentielle à la créativité mathématique ${ }^{8}$.

Ainsi que le note l'auteure, introduire le mot «collection» n'est pas seulement créer un mot ou un concept nouveau, c'est produire l'entité collection, entité réelle au sens où les troisièmes (selon les trois catégories logico-ontologiques de Peirce) le sont, les troisièmes correspondant aux régularités et aux universaux. Mais Peirce reconnaît que, parmi les abstractions, certaines sont fictives et certaines sont réelles (CP 4.549), et qu'il faut trouver un critère pour les départager.

Christiane Chauviré reproche à Peirce de ne pas avoir expliqué la différence qu'il semble pourtant bien y avoir, au sein des troisièmes, entre une entité comme la dureté, qui est une disposition inscrite dans la nature physique de certains objets matériels, donc radicalement indépendante de nous, et une entité comme la collection, produit de notre spéculation. La dureté et la collection sont-elles réelles au même titre, et ne faudrait-il pas distinguer différents types de troisièmes?

Il eut été fort intéressant que l'auteure confronte ses réserves sur la possibilité d'appliquer le pragmatisme aux entités mathématiques, avec la défense du pragmatisme peircien, en philosophie des mathématiques, par Gerhardt Heinzmann dans «L'épistémologie mathématique de Gonseth dans la perspective du pragmatisme de Peirce» (Dialectica, 1990). Heinzmann a montré que Peirce a fait bien plus que simplement utiliser en mathématique une maxime adaptée pour comprendre la réalité des propriétés de la physique. Il a analysé en particulier comment Peirce (CP 5.3) rejette l'objection que sa maxime serait incompatible avec la doctrine mathématique des incommensurables qui, par nature, ne peut pas être établie par des mesures physiques.

On trouve également chez Pietarinen, «Which Philosophy of Mathematics is Pragmaticism ?" ", une défense du pragmatisme peircien en philosophie des mathématiques, selon lequel toute découverte scientifique, même mathématique, repose sur l'observation (ce qui n'est pas de l'empirisme ni même du quasi-empiricisme (Lakatos, Putnam), mais se compare plutôt avec la notion de variation eidétique chez Husserl).

D'après Peirce, les mathématiques ne peuvent progresser que par une pratique spécifique, une certaine manipulation de signes: «Ce n'est pas par une simple contemplation mentale ou par un effort de vision mentale. C'est en manipulant sur du papier ou dans l'imagination des formules ou d'autres diagrammes - en expérimentant sur eux...» (CP 4.86). Christiane Chauviré

8. Au passage, Peirce a élaboré une logique du second ordre (CP 4.83).

9. Mathew Moore (dir.), New Essays on Peirce's Mathematical Philosophy, Open Court, à paraître. 
propose un parallèle stimulant entre Wittgenstein et Peirce pour soutenir que la pratique effective des mathématiques devrait être au cœur de la philosophie des mathématiques: "Souvenons-nous qu'en mathématiques les signes mêmes font la mathématique, et non décrivent la mathématique "; «on ne peut écrire la mathématique, on peut seulement la faire » (Wittgenstein, Remarques philosophiques, $\mathbb{S} 157$ et $\mathbb{S} 159$ ). L'auteure rappelle avec justesse qu'il y a là une différence fondamentale par rapport à Frege, lequel a été détourné de l'importance de la pratique mathématique par son platonisme: "En traçant une droite, nous prenons simplement conscience de ce qui existe indépendamment de nous » (Frege, Naschlass, p. 246-247).

Comme le fait remarquer C. Chauviré, chez Peirce, la signification des signes mathématiques ne repose pas sur leur concevabilité, mais sur leur valeur opérationnelle (CP 4.133, 1893); d'où une certaine proximité avec le formalisme hilbertien. Peirce et Hilbert partagent non seulement une mutuelle admiration pour Kant, mais, de plus, comme le montre Peckhaus, "The Pragmatism of Hilbert's program» (Synthèse, 2003), dans son étude des systèmes de signes, Hilbert fait appel à Schröder qui lui-même se fonde sur Peirce.

L'auteure insiste particulièrement sur l'importance de la distinction analytique/synthétique, notamment dans le cadre de la controverse sur l'origine de la fécondité des mathématiques. Comme l'a souligné Poincaré, il y a ici une difficulté fondamentale: comment les mathématiques peuvent-elles être à la fois rigoureuses et fécondes si la rigueur du raisonnement suppose sa stérilité ? Christiane Chauviré rappelle que, au début du $\mathrm{Xx}^{\mathrm{e}}$ siècle, dans le sillage de Kant et de sa distinction analytique/synthétique, on peut distinguer deux courants principaux en philosophie des mathématiques. Celui qui s'oppose fortement à Kant, de loin le plus dominant des deux, est celui auquel peuvent se rattacher Weierstrass, Dedekind et Russell. Ce premier courant est marqué par la recherche croissante de la rigueur, par la crainte des dangers de l'intuition en mathématique, et par la thèse selon laquelle les raisonnements mathématiques ne sont pas synthétiques a priori, mais analytiques. La position de Frege - plus nuancée concernant la nature des raisonnements géométriques - , est néanmoins proche de ce premier courant, car Frege soutient que la fécondité des mathématiques s'explique à partir du contenu des définitions, et non à partir des constructions des mathématiciens.

Le second courant, nettement moins dominant au début du $\mathrm{xx}^{e}$ siècle, est celui qui demeure assez proche du point de vue kantien, et auquel on peut rattacher Peirce et Poincaré, malgré leurs très grandes divergences de vue sur d'autres questions philosophiques. Ainsi, Poincaré affirme que la fécondité de l'arithmétique ne repose pas sur des démonstrations analytiques (stériles selon lui), mais sur ce qu'il appelle le principe d'induction mathématique. Plus récemment, la position d'Hintikka poursuit et développe ce second courant en rejetant l'idée selon laquelle la formalisation des mathématiques dispenserait du recours aux constructions qui seraient superflues. 
Au lieu de reprendre la distinction kantienne analytique/synthétique, Peirce élabore (dans ses années de maturité) un couple conceptuel plus adapté à l'étude du raisonnement mathématique : le couple corollariel/théorématique. Une déduction est "corollarielle lorsque, pour percevoir immédiatement que la conclusion est valide dans le cas étudié, il suffit d'imaginer tous les cas pour lesquels les prémisses sont vraies» (NEM 4.38); une déduction est théorématique lorsque pour faire apparaître la conclusion il est nécessaire de faire une construction supplémentaire sur «le diagramme de la vérité des prémisses» (NEM 3.869, 1909).

Cependant, comme le fait justement remarquer Christiane Chauviré, Peirce est obligé d'admettre que des théorèmes deviennent, selon son critère, des corollaires "par suite d'une amélioration dans le système de logique " (CP 4.613), mais il distingue la tâche du mathématicien, qui démontre les théorèmes, et la tâche du logicien, qui analyse les démonstrations et les «mouline [...] pour en faire des corollaires» (NEM 4.289).

L'auteure soutient qu'avec la logique polyadique, la maîtrise de ce nouveau couple conceptuel permet d'éviter certaines erreurs kantiennes. Ainsi, dans NEM 4.58-59, Peirce utilise le couple corollariel/théorématique et la logique des relatifs, pour affirmer que Kant s'est trompé en soutenant que $7+5=12$ est un jugement synthétique a priori. En utilisant la définition de «+» et la notion de successeur immédiat, Peirce montre qu'on n'a pas besoin de raisonnement théorématique pour prouver, à partir des définitions, que « $7+5=12$ ».

Comme le fait remarquer Hintikka, les problèmes liés à l'omniscience logique sont résolus par la distinction corollariel/théorématique, car il n'y a rien d'étonnant à ce que quelqu'un échoue à tirer une conclusion théorématique, et il n'y a ainsi pas de lien direct entre la connaissance parfaite des prémisses et l'accomplissement d'une déduction théorématique.

Mais pourquoi cette distinction centrale chez Peirce est-elle restée pratiquement inaperçue chez les grands logiciens $\mathrm{du} \mathrm{xx}^{\mathrm{e}}$ siècle avant Hintikka? L'auteure explique que cette distinction est incompatible avec le courant dominant en logique, au début du $\mathrm{xx}^{\mathrm{e}}$ siècle, qui rejette le rôle des «intuitions " et insiste sur le caractère essentiellement symbolique de la logique, alors que Peirce insiste au contraire sur la nécessité de considérer également, outre les aspects symboliques, les aspects iconiques et indexicaux dans nos inférences logiques.

Christiane Chauviré soutient une thèse interprétative originale et très intéressante selon laquelle, bien que Peirce ne le dise jamais explicitement, il y aurait deux concepts d'analycité chez Peirce: une analycité épistémique (le corollariel) et une analycité logique, assez proche de l'analytique chez Frege, qui correspond au déductivement démontrable. Selon cette interprétation, chez Peirce, tous les corollaires seraient analytiques d'un point de vue épistémique, et analytiques d'un point de vue logique, alors que tous les théorèmes seraient synthétiques d'un point de vue épistémique, et analytiques 
d'un point de vue logique. D'après l'auteure, et je partage son avis, le bénéfice de la distinction qu'elle introduit entre l'analytique mathématique (corollariel) et l'analytique logique, c'est qu'elle autorise à soutenir que, pour Peirce, les inférences et les énoncés mathématiques peuvent être à la fois analytiques (d'un point de vue logique) et synthétiques (d'un point de vue épistémique).

Elle fait remarquer que c'est l'attention portée par Peirce à la pratique effective des mathématiques qui lui a permis d'élaborer un critère précis de la synthéticité en mathématique: l'introduction d'entités auxiliaires qui disparaissent dans la formulation finale du théorème démontré. Cela permet à Peirce d'expliquer clairement comment une formule mathématique peut être dite à la fois analytique (démontrable), et synthétique.

On peut regretter que l'auteure ne compare pas sa thèse avec d'autres interprétations récentes, par exemple celle de Levy, qui soutient qu'il faut distinguer trois niveaux d'analycité chez Peirce, dont deux correspondent en gros à ceux distingués par Christiane Chauviré, et où le troisième correspond à un raisonnement ayant un "faible degré» de théorématicité (où l'idée nouvelle est en quelque sorte dérivable à partir des prémisses); (voir Stephen Levy, «Peirce's Theoremic/Corollarial Distinction and the Interconnections between Mathematics and Logic", Studies in the Logic of C. S. Peirce, 1997).

Sans doute influencée par l'interprétation d'Hintikka ( C.S. Peirce's "First Real Discovery" and Its Contemporary Relevance», The Monist, 1980), l'auteure ramène souvent la distinction corollariel/théorématique à la distinction trivial/non trivial, ce qui à mon avis ne rend pas assez justice à la subtilité de la distinction. D'ailleurs, Daniel Campos («The Imagination and Hypothesis-Making in Mathematics: A Peircean Account $\left.{ }^{10}\right)$, a montré qu'une inférence corollarielle pouvait ne pas être triviale. Tout comme Hintikka, C. Chauviré n'a pas bien vu qu'une déduction corollarielle pouvait exiger un effort d'imagination qui n'est ni mécanique ni trivial.

L'auteure aurait dû faire le lien entre la notion de corollaire et les courtes mais intéressantes pages qu'elle consacre à l'évidence mathématique, décrite par Peirce comme étant analogue au jugement de perception «à ceci près qu'au lieu de se référer au percept que nous impose la perception, il se réfère à une création de notre imagination » (CP 7.659). Peirce a d'ailleurs développé une phénoménologie de la perception mathématique (NEM 4.316-7).

Christiane Chauviré se demande comment Peirce peut, d'une part, affirmer que c'est fondamentalement par une icône (ou plus précisément par un diagramme comportant des éléments indexicaux et symboliques l'aidant à remplir sa fonction iconique) que s'impose à nous la nécessité mathématique (CP 4.532), tout en affirmant, d'autre part, que contrairement à

10. Dans Mathew Moore (dir.), New Essays on Peirce's Mathematical Philosophy, Open Court, à paraître, p. 25. 
l'indice qui tient lieu de ceci ou cela, l'icône ne peut pas renvoyer à l'existence, mais à la possibilité. Christiane Chauviré reproche à Peirce de faire reposer la nécessité mathématique sur l'expérience du mathématicien, alors que, selon elle, l'observation du raisonnement, centrale pour Peirce (CP 2.605), est insuffisante à garantir la nécessité.

C'est une objection intéressante, toutefois il me semble que Peirce a de solides arguments contre. Il aurait sans doute répondu, premièrement, que l'esprit n'est pas un sujet solipsiste isolé, mais une réalité collective (MS 18, 1907) ${ }^{11}$; deuxièmement, que l'expérience mathématique est générique et contient en elle une infinité d'expériences semblables; troisièmement, que contrairement aux cas des faits du monde, nous sommes compétents pour établir un lien de nécessité entre des hypothèses mathématiques, parce que nous les créons nous-mêmes (CP 2.191). Sur la question de savoir si le faillibilisme de Peirce s'étend ou non aux mathématiques, voir Elizabeth Cooke, "Peirce, Fallibilism, and the Science of Mathematics", Philosophia Mathematica, 2003.

Les commentateurs s'accordent mal sur la question du rapport de Peirce au logicisme, bien que la plupart admettent, comme Christiane Chauviré, que Peirce n'est pas logiciste sur le fond, tout en ayant de la sympathie pour certaines thèses logicistes, notamment en soutenant que tous les énoncés mathématiques sont analytiques. La position de Peirce est incompatible avec le logicisme, car il affirme très souvent que les mathématiques, en tant que science hypothético-déductive reposant sur la logica utens, sont la seule science indépendante de la logique (logica docens), bien qu'il reconnaisse, surtout pour certaines branches récentes des mathématiques - comme celles traitant de l'infini (NEM 4.xxiii), des infinitésimaux ou de la topologie -, qu'une analyse logique serait très utile à leurs progrès.

Pour conclure, Christiane Chauviré éclaircit de nombreux aspects importants de la philosophie des mathématiques de Peirce. Mais certains aspects ne sont pas ou très peu abordés, notamment tout ce qui est lié à la théorie des ensembles, à l'infini mathématique et aux divers modes de raisonnement qui y sont applicables, aux infinitésimaux, à la continuité et à la topologie. Il eut été éclairant pour le lecteur de pouvoir plus précisément situer Peirce, non seulement par rapport au kantisme, au logicisme, au formalisme, au platonisme et à l'empirisme, mais aussi par rapport à l'intuitionnisme, au structuralisme, au quasi-empiricisme ainsi qu'à l'égard de la philosophie de Husserl. On peut aussi regretter que l'auteure ne confronte pas assez ses interprétations avec la littérature secondaire récente, qu'elle n'explicite pas assez l'évolution de la pensée de Peirce sur des thèmes majeurs comme l'ontologie ou l'interprétation de Kant, et qu'elle n'entre jamais dans

11 Microfilm Edition selon Richard S. Robin, Annotated Catalogue of the Papers of Charles S. Peirce, Amherst, The University of Massachusetts Press, 1967. 
l'analyse des théories mathématiques, alors que l'étude de la pratique mathématique est essentielle pour Peirce.

Le mérite principal de cet ouvrage est, à mon avis, de proposer sans doute la meilleure analyse comparative jamais réalisée de la philosophie des mathématiques de Peirce par rapport à celles de Kant, Frege, Wittgenstein et Hintikka, tout en apportant des éclaircissements très intéressants pour situer Peirce par rapport au platonisme mathématique.

JÉRÔME HAVENEL

Université du Québec à Montréal

\begin{abstract}
Anne-Marie Décaillot, Cantor et la France. Correspondance du mathématicien allemand avec les Français à la fin du XIX siècle, Paris, Éditions Kimé, 2008, 347 p.
\end{abstract}

L'ouvrage de Décaillot est construit autour des chapitres suivants: 1. Les sources; 2. Les combats de Cantor; 3. Des intellectuels catholiques aux occultistes. Une étonnante diversité de rélations; 4. À la recherche d'une harmonie entre science et foi (théologie, philosophie et mathématique); 5. Cantor et la conjecture de Goldbach; 6 . Est beau ce qui est beau intérieurement ; 7. La correspondance. L'ouvrage comporte également quatre appendices: 1. Liste des lettres de Cantor aux Français; 2. Les différents sens de l'expression «infini mathématique»; 3 . La genèse de la théorie cantorienne des ensembles; 4 . Calculs probabilistes en théorie des nombres. Il peut être conseillé de procéder d'abord à la lecture des lettres (ce qui peut aisément se faire d'un seul coup si on ne les lit que dans une seule langue), pour ensuite se tourner vers les chapitres thématiques détaillés de l'auteur pour de l'information d'arrière-plan.

Les trente-sept lettres et esquisses composant cet ensemble sont présentées dans un ordre chronologique et s'étendent sur une période de dix ans, entre 1886 et 1896, commençant ainsi à une période où Cantor avait réalisé la plus grande partie de son travail en théorie des ensembles, tout juste après sa première dépression en 1884 , et jusqu'un peu avant sa deuxième dépression de 1899. Toutes les lettres sont de Cantor, à l'exception d'une qui lui est adressée.

Ses quatorze correspondants sont les mathématiciens Charles Hermite, Camille Jordan, Henri Poincaré, Jules Tannery, les intellectuels catholiques Élie Blanc, Maurice d'Hulst, Claude Alphonse Valson; les polytechniciens Charles-Ange Laisant et Émile Lemoine; le philosophe et historien des sciences Paul Tannery: l'occultiste Papus (Gérard Encausse); le scientifique Charles Henry, le sénateur Barthélémy Saint-Hilaire, ainsi que le fondateur de la Revue de métaphysique et de morale, Xavier Léon. 